\title{
Intraorbital wooden foreign body - a case report
}

\author{
Piotr Kanclerz ${ }^{1}$, Agnieszka Chrząszcz-Włodarczyk² ${ }^{2}$ Andrzej Grzybowski ${ }^{3,4}$ \\ ${ }^{1}$ Hygeia Clinic, Department of Ophthalmology, Gdansk, Poland \\ ${ }^{2}$ Department of Ophthalmology, Medical University of Gdansk, Poland \\ ${ }^{3} /$ nstitute for Research in Ophthalmology, Foundation for Ophthalmology Development, Poznan, Poland \\ ${ }^{4}$ Department of Ophthalmology, Medical University of Warmia i Mazury, Olsztyn, Poland
}

\begin{abstract}
BACKGROUND: Wood as an intraorbital foreign body (IOFB) is infrequent, and as it is organic matter patients represent an increased risk of infection. The aim of this study is to report the complexity of treatment of patient with a wooden IOFB. We describe a case of a 67 -year-old male with a wooden IOFB.

CASE REPORT: On referral, the patient presented with exophthalmos, pain, blurry vision and discharge from his left eye was admitted to the Department of Ophthalmology, Medical University of Gdańsk. The previous evening, he fell with his face down. Computed tomography revealed a low-density IOFB of approximately -980 Hounsfield Units, sized $62 \times 8 \mathrm{~mm}$, in the area of left orbit, ethmoid and sphenoid sinus. Subsequently, the IOFB was removed under general anaesthesia. After three days of empirical antibiotic therapy, the patient was discharged with a switch to oral antibiotics. Shortly after the conversion to oral therapy, he developed an orbital inflammatory syndrome and was readmitted to the hospital. His condition improved after readministration of parenteral antibiotics.

CONCLUSION: This case demonstrates that wooden IOFBs should be treated with caution. Parenteral antibiotic delivery leads to higher serum levels than with oral intake, and in this case, was essential in preventing infection after IOFB removal.
\end{abstract}

KEY WORDS: foreign body; wood; orbit; computed tomography

Ophthalmol J 2020; Vol. 5, 51-54

\section{INTRODUCTION}

One out of every six cases of penetrating orbital trauma is associated with an intraorbital foreign body (IOFB) [1]. Intraorbital foreign bodies might present a wide range of imaging manifestation, and the entry wound may often be small and self-sealing, resulting in diagnostic difficulties. The aim of this study is to report the complexity of treatment of a patient with a wooden IOFB.

\section{CASE REPORT}

A 67-year-old Caucasian male presented to the Department of Ophthalmology of the Medical University of Gdańsk with decreased to hand movements visual acuity in the left eye, along with diplopia, exophthalmos and severe limitation of eye movements in all directions (Fig. 1A). The previous evening, he fell with his face down. He did not report any general diseases or prior antibiotic 


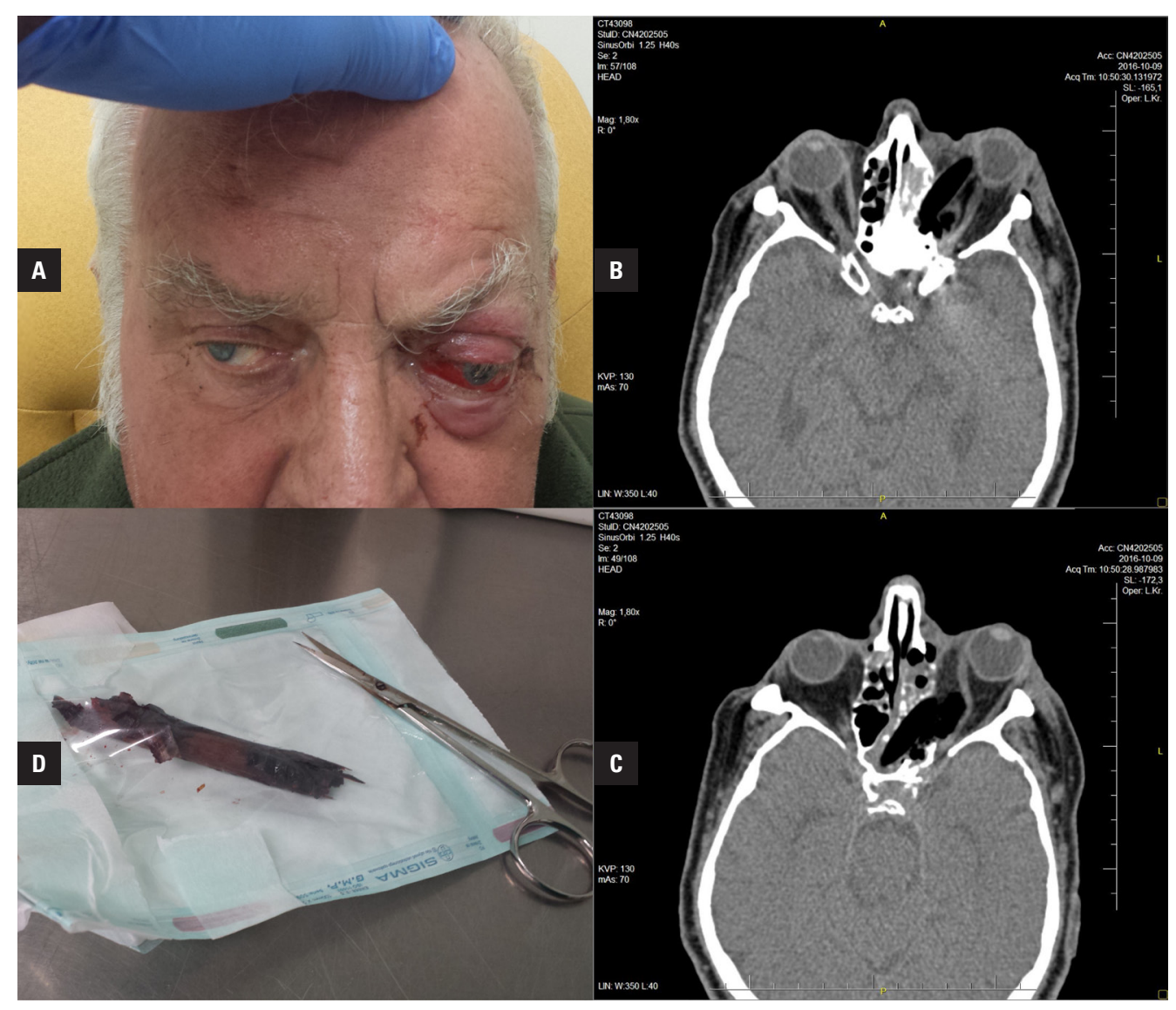

FIGURE 1. A. The patient preoperatively. He was asked to look right; a significant limitation in eye movements, pain, severe exophthalmos and discharge from the left eye is noted. B-C. Computed tomography revealed a low-density foreign body of approximately -980 Hounsfield Units (HU), sized $62 \times 8 \mathrm{~mm}$ in the area of left orbit, ethmoid and sphenoid sinus. D. A macroscopic image of the foreign body just after removal. For size comparison, iris scissors are presented

use. Computed tomography revealed a low-density foreign body of approximately -980 Hounsfield Units (HU), sized $62 \times 8 \mathrm{~mm}$ in the area of left orbit, ethmoid and sphenoid sinus (Fig. 1B, C). The intraocular pressure of the left eye was palpably increased, the pupil was large and poorly reactive, no entry wound was found. Systemic therapy with intravenous cefuroxime $750 \mathrm{mg}$ three times daily (TID), metronidazole $500 \mathrm{mg}$ TID and topical tobramycin $0.3 \%$ and dexamethasone $0.1 \%$ was prescribed. The patient received enoxaparin $80 \mathrm{mg} / 0.8 \mathrm{~mL}$ subcutaneously and a tetanus immune globulin $250 \mathrm{U}$ IM. As magnetic resonance imaging (MRI) was not available in a reasonable timespan, a surgical intervention in general anesthesia was carried out.

The surgery was performed by the ENT specialist; after anesthetization, a $1.5 \mathrm{~cm}$-long wound was noted in the medial angle of the upper left eyelid with the edge of the IOFB visible in it. An anterior orbitotomy was performed in the extension of lacer- ation, and after scrupulous tissue dissection around the IOFB the wooden piece was removed (Fig. 1D). Swabs were taken and several additional splinters were removed with copious irrigation of the cavity using a 3\% hydrogen peroxide solution [2]. The eye was reviewed showing no signs of discontinuity of the sclera. Intraoperative tonometry revealed a pressure of $3 \mathrm{~mm} \mathrm{Hg}$ in the left eye, with no signs of scleral discontinuity.

In the postoperative MRI, the medial rectus muscle (MRM) was thickened with the suspicion of a small $10-\mathrm{mm}$ splinter within the muscle. Nevertheless, the local condition improved. Two days after surgery the patient demonstrated the visual acuity in his left eye of 0.1 , minor exophthalmos and eyelid edema, and was discharged on empirical antibiotic therapy. A sequential switch to cefuroxime acetyl $500 \mathrm{mg}$ twice daily and metronidazole $250 \mathrm{mg}$ TID orally was recommended. Topical tobramycin $0.3 \%$ and dexamethasone $0.1 \%$ four times daily was to be continued. 


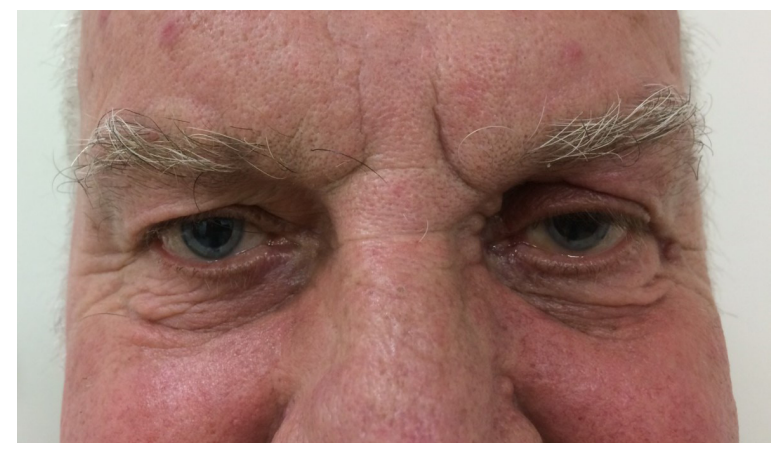

FIGURE 2. One month after surgery the patient presented in his left eye minimal exophthalmos and only partial limitation of eye movements medially

The next day he was readmitted to the emergency unit because of increasing exophthalmos and pain in his left eye and orbital region. In contrast-enhanced MRI MRM remained thickened with no signs of a splinter. Cavernous sinus thrombosis was excluded. The results of intraoperative swabs revealed Escherichia coli, Lecleria adecarboxylata, Bacillus cereus and coagulase-negative staphylococcus (CNS). All of the microbes were found to be susceptible to cefuroxime; however, the susceptibility of $B$. cereus was not assessed. As no anaerobic bacteria were found, susceptibility to metronidazole was not analyzed. Intravenous treatment including dexamethasone $12 \mathrm{mg}$ once daily was administered, while cefuroxime with metronidazole was reintroduced. Additionally, intramuscular galantamine hydrobromide $2.5 \mathrm{mg}$ and combined $100 \mathrm{mg}$ thiamine, $100 \mathrm{mg}$ pyridoxine and $1 \mathrm{mg}$ cyanocobalamin were recommended in order to relieve the pain and enhance nerve regeneration. The patient improved over the following seven days.

One month after surgery he presented minimal exophthalmos and partial limitation of eye movement medially (Fig. 2). Since it was attributed to the medial rectus muscle injury no additional treatment was recommended.

\section{DISCUSSION}

The current literature reports that the diagnosis and management of IOFBs might be contentious. Particularly wooden IOFBs can be occult due to late presentation and the lack of external signs of injury [1]. In the study by Shelsta et al., the time from injury to the presentation was highly variable, with a mean of 62 days from the injury to the presentation (range 1-17 months; median
3 days and only $43 \%$ of cases presented within 24 hours of injury) [1]. In other studies, the time from injury to admission was up to 22 months [ 1 , $3-5]$. One study found that the time from injury to the presentation was negatively correlated with the size of the wooden IOFB [4]. As wood presents a wide range of $\mathrm{CT}$ densities, the imaging findings in these cases are diverse [3]; in a single study, an orbital foreign body after the CT scan was suggested only in six out of eleven cases [6]. The CT densities vary between $-984 \mathrm{HU}$ for dry porous wood, $-70 \mathrm{HU}$ for wet wood, and up to $+156 \mathrm{HU}$ for tree bark encapsulated with soft tissue [7-9]. Performing MRI scans is beneficial when the wood is surrounded by fat or extracellular fluid, although MRI was not proven to be more useful than CT in wooden IOFBs [10]. In the study by $\mathrm{Li}$ et al. the IOFB was not found during surgery in three out of 11 cases, while in two cases fistula tracts have developed due to late removal [6]. Due to its fragile structure, complete removal of a wooden foreign body might be impossible, and the remaining splinters are a potential source of infection. Complications include abscess formation, local infection, development of a fistula or granuloma, and these problems might develop several months or years after successful IOFB removal [1, 4, 11].

Infections associated with organic foreign bodies occur in up to $64 \%$ of IOFBs, even if antibiotics are applied [1]. The porous structure of wood and its characteristics of organic matter support bacterial ingrowth. Pathogens commonly cultured such foreign bodies include Streptococcus sp., E. coli, CNS and anaerobes [1]. In our case, four types of bacteria were isolated from the wound. Two ubiquitous species, which are susceptible to several antimicrobials including cefuroxime were the CNS and Leclercia adecarboxylata. The former is a gram-negative bacteria, a member of the family Enterobacteriaceae, and has a role in infections mainly in immunocompetent patients [12]. The third species, B. cereus is a soil bacterium and can be found on plants; it could an incidental finding. On the other hand, B. cereus manifest resistance to $\beta$-lactam antibiotics [13]; in our case, it was not possible to determine its susceptibility. Finally, E. coli is commonly resistant against routinely used antibiotics; one of the most important determinant for resistance is the recent antibiotic use [14]. In this subject, the strain was susceptible to cefuroxime. Additional therapy with ciprofloxacin to which $B$. cereus is highly susceptible could have been considered [13]. 
In general, intravenous administration of antibiotics leads to higher serum levels than with oral intake [15]. However, conversion to oral therapy has many advantages, including less healthcare costs, fewer complications associated with parenteral therapy and earlier hospital discharge [16]. The poor outcome of oral therapy in the described case could be presumably attributed to insufficient therapeutic levels of oral treatment. This report demonstrates that wooden IOFBs should be treated with caution; in this case, parenteral antibiotic delivery was essential in preventing a local infection after IOFB removal.

\section{Acknowledgements}

Nothing to acknowledge.

\section{Conflict of interests and funding}

No conflicting relationship exists for any author. P.K. reports non-financial support from Visim. A.Ch.W. has nothing to disclose. A.G. reports grants, personal fees and non-financial support from Bayer; grants, non-financial support from Novartis; non-financial support from Alcon, personal fees and non-financial support from Valeant, grants and non-financial support from Allergan, grants and non-financial support from Pfizer, grants, and financial support from Santen.

\section{REFERENCES}

1. Shelsta HN, Bilyk JR, Rubin PAD, et al. Wooden intraorbital foreign body injuries: clinical characteristics and outcomes of 23 patients. Ophthalmic Plast Reconstr Surg. 2010; 26(4): 238-244, doi: 10.1097/ IOP.0b013e3181bd7509, indexed in Pubmed: 20523259.

2. Malik N. Benign Tumors of the Jaw Bones. In: Malik N. ed. Textbook of Oral and Maxillofacial Surgery. Jaypee Brothers Medical Pub 2016: 674-734.

3. Zhou L, Li SY, Cui JP, et al. Analysis of missed diagnosis of orbital foreign bodies. Exp Ther Med. 2017; 13(4): 1275-1278, doi: 10.3892/ etm.2017.4146, indexed in Pubmed: 28413466.
4. Taş S, Top H. Intraorbital wooden foreign body: clinical analysis of 32 cases, a 10-year experience. Ulus Travma Acil Cerrahi Derg. 2014; 20(1): 51-55, doi: 10.5505/tites.2014.93876, indexed in Pubmed: 24639316.

5. John SS, Rehman TA, John D, et al. Missed diagnosis of a wooden intra-orbital foreign body. Indian J Ophthalmol. 2008; 56(4): 322-324, doi: 10.4103/0301-4738.41416, indexed in Pubmed: 18579994.

6. Li J, Zhou LP, Jin J, et al. Clinical diagnosis and treatment of intraorbital wooden foreign bodies. Chin J Traumatol. 2016; 19(6): 322-325, doi: 10.1016/j.cjtee.2016.04.006, indexed in Pubmed: 28088934.

7. van der Wal KG, Boukes RJ. Intraorbital bamboo foreign body in a chronic stage: case report. Int J Oral Maxillofac Surg. 2000; 29(6): 428-429, doi: 10.1034/j.1399-0020.2000.290606.x, indexed in Pubmed: 11202322.

8. Glatt $\mathrm{H}$, custer $\mathrm{p}$, Barrett $\mathrm{L}$. Magnetic Resonance Imaging and COmputead Tomography in a Model of wooden Foreign Bodies in the orbit. Ophthal Plast Reconstruct Surg. 1990; 6(4): 290, doi: 10.1097/00002341-199012000-00036.

9. Krimmel M, Cornelius CP, Stojadinovic $S$, et al. Wooden foreign bodies in facial injury: a radiological pitfall. Int J Oral Maxillofac Surg. 2001; 30(5): 445-447, doi: 10.1054/ijom.2001.0109, indexed in Pubmed: 11720049.

10. McGuckin JF, Akhtar N, Ho VT, et al. CT and MR evaluation of a wooden foreign body in an in vitro model of the orbit. AJNR Am J Neuroradiol. 1996 Jan; 17(1): 129-33, indexed in Pubmed: 8770263.

11. Nishio $Y$, Hayashi $N$, Hamada $H$, et al. A case of delayed brain abscess due to a retained intracranial wooden foreign body: a case report and review of the last 20 years. Acta Neurochir (Wien). 2004; 146(8): 847-850, doi: 10.1007/s00701-004-0283-7, indexed in Pubmed: 15254807.

12. Spiegelhauer MR, Andersen PF, Frandsen TH, et al. Leclercia adecarboxylata: a case report and literature review of 74 cases demonstrating its pathogenicity in immunocompromised patients. Infect Dis (Lond). 2019; 51(3): 179-188, doi: 10.1080/23744235.2018.1536830, indexed in Pubmed: 30488747.

13. Fiedler G, Schneider C, Igbinosa EO, et al. Antibiotics resistance and toxin profiles of Bacillus cereus-group isolates from fresh vegetables from German retail markets. BMC Microbiol. 2019; 19(1): 250, doi: 10.1186/s12866-019-1632-2, indexed in Pubmed: 31706266.

14. Duerink DO, Lestari ES, Hadi U, et al. Study Group Antimicrobial Resistance in Indonesia: Prevalence and Prevention (AMRIN). Determinants of carriage of resistant Escherichia coli in the Indonesian population inside and outside hospitals. J Antimicrob Chemother. 2007; 60(2): 377-384, doi: 10.1093/jac/dkm197, indexed in Pubmed: 17595290.

15. Enenkel S, Stille W. Administration of AntiBiotics. Antibiotics in the Tropics. 1988: 39-40, doi: 10.1007/978-3-642-73276-8 5 .

16. Shrayteh ZM, Rahal MK, Malaeb DN. Practice of switch from intravenous to oral antibiotics. Springerplus. 2014; 3: 717, doi: 10.1186/21931801-3-717, indexed in Pubmed: 25674457. 